\title{
Evolution of Atom Probe Data Collection Toward Optimized and Fully Automated Acquisition
}

Ty J. Prosa ${ }^{1}$, David A. Reinhard ${ }^{1}$, H.G. François-Saint-Cyr ${ }^{1}$, Isabelle Martin ${ }^{1}$, Katherine P. Rice ${ }^{1}$, Yimeng Chen ${ }^{1}$ and David J. Larson ${ }^{1}$

1. CAMECA Instruments, Inc., 5470 Nobel Drive, Madison, WI 53711 USA.

Atom Probe Tomography (APT) has come a long way since the invention of the atom probe by Müller, Panitz and McLane in 1967 [1]. The state of the art then was to collect some tens of atoms per hour and record oscilloscope traces on film to measure time-of-flight (Figure 1). Today, modern electronics easily allow for collection rates of greater than 100 million ions per hour (limited primarily by specimen survivability rather than instrumentation) with software capable of parallel conversion from time-offlight to mass-to-charge with self-consistent estimates for corrections due to variations in voltage and detector impact position [2]. As application of APT continues to evolve and grow to take on new material characterization challenges, time-to-knowledge becomes more critical, especially to meet the requirements for users like those in the semiconductor industry. Improvements such as automated sample positioning and start-up, coupled with smarter acquisition algorithms and recipes, are necessary to provide full tool utilization, along with optimized yield and data quality to achieve minimum time-toknowledge.

Our group has been working to understand how various analysis parameters affect yield and data quality on internal standards with the goal of incorporating this knowledge into acquisition protocols. Live metrics, such as charge-state ratios (CSRs), can provide information related to the evaporation field during analysis. By monitoring this metric, the applied evaporation field can be manipulated to provide improved specimen survival and higher acquisition rates which leads to shorter time-to-knowledge and often improved data quality as well. Understanding the relationship between CSR and electric field beyond theoretical predictions [3] can also affect data quality by enabling more accurate reconstructions (improved spatial accuracy).

We have studied multiple internal standards containing silicon and silicon oxide. Figure 2 highlights some results of these efforts. Even though these data are all silicon CSRs, the trends and relative field values are different depending on whether these ions originated from the silicon or oxide regions. We note that a strictly post-ionization explanation of observed CSRs [3] is insufficient to explain the discrepancy. In fact, for silicon post-ionization, it should not be possible to observe all three silicon charge states simultaneously. This suggests that the evaporation physics for the oxide is different than for pure silicon and may have multiple physical processes taking place as part of the evaporation [4].

In this presentation, we discuss and highlight some factors one might consider when optimising analysis conditions, especially toward fully automated acquisition. Understanding the detailed trade-offs between yield, data quality, and time-to-knowledge is necessary to make appropriate decisions about analysis conditions. While wider adoption of the technique has drastically increased the use of APT to characterize materials, detailed study to better understand the physics of analysis may be waning. More systematic work needs to be done to map the analysis parameter space for new materials to take full advantage of modern APT. 


\section{References:}

[1] E.W. Müller, J.A. Panitz and S.B. McLane, Rev. Sci. Instrum. 39 (1968) p. 83.

[2] D.J. Larson et al., "Local Electrode Atom Probe Tomography", (Springer, New York) (2013).

[3] D.R. Kingham, Surf. Sci. 116 (1981) p. 273.

[4] D.K. Schreiber et al., Appl. Phys. Lett. 105 (2014) p. 244106.

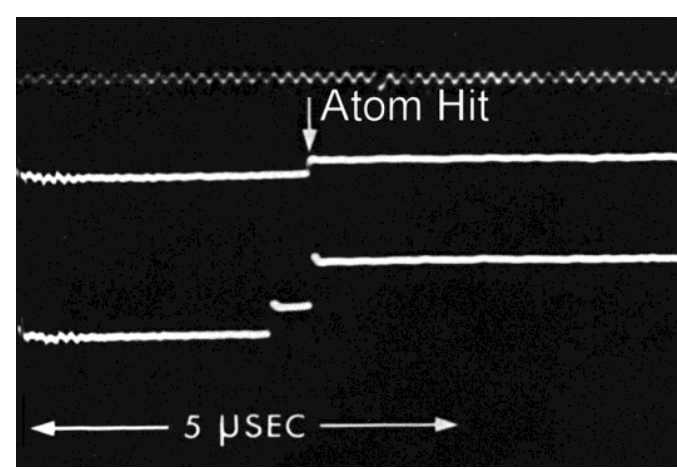

Figure 1. Photograph of an oscilloscope screenshot with two traces. The lower trace shows two atom hits. The sine wave at the top was used as a known time base (figure courtesy J.A. Panitz).

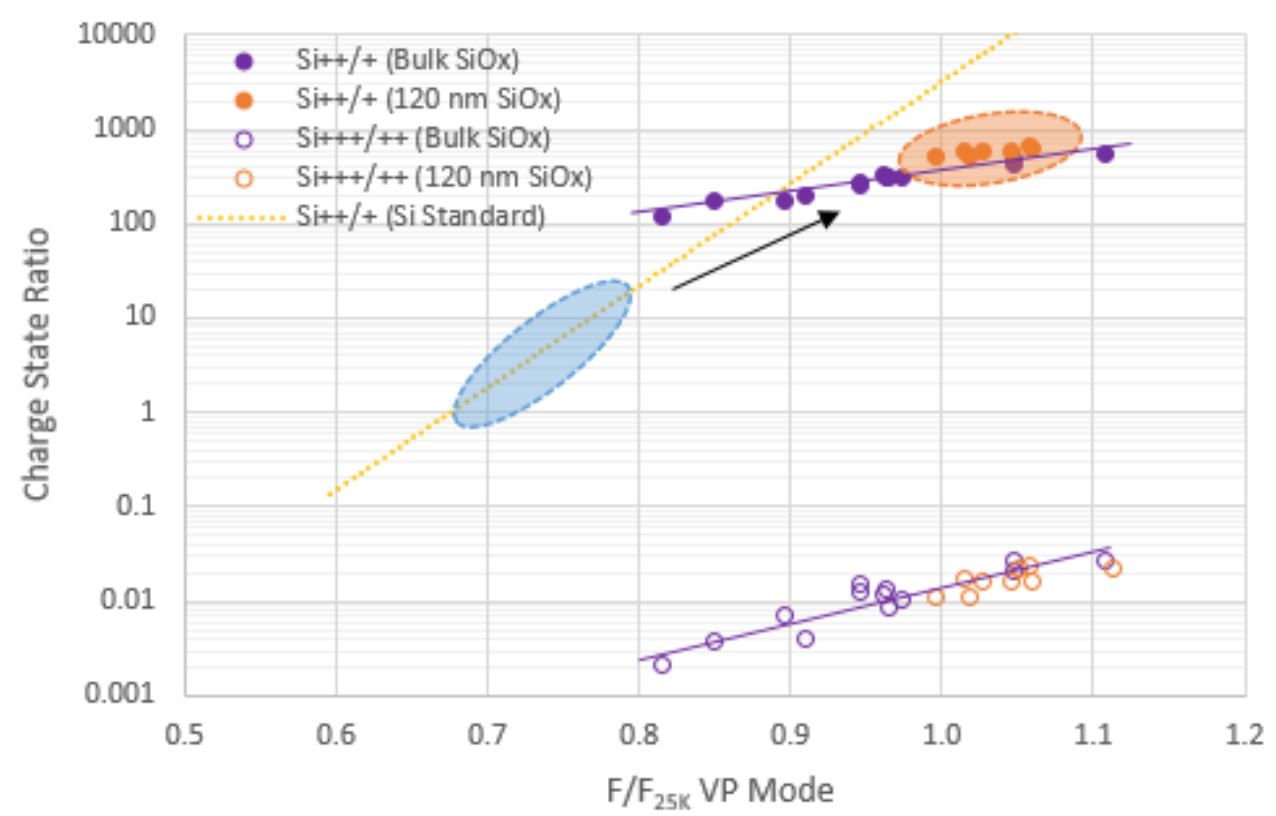

Figure 2. Silicon charge-state-ratios $\left(\mathrm{Si}^{++} / \mathrm{Si}^{+}\right.$and $\left.\mathrm{Si}^{+++} / \mathrm{Si}^{++} \mathrm{CSRs}\right)$ and relative field estimates for $\mathrm{SiOx}$ based on measurements of pure $<100>\mathrm{Si}$ (gold-dotted line). The fields are relative to the field required to evaporate silicon in voltage-pulse mode with $20 \%$ pulse fraction at $25 \mathrm{~K}$. A $120 \mathrm{~nm}$, thermally grown $\mathrm{SiOx}$ from <100> Si substrate was analyzed with a LEAP $5000 \mathrm{XS}$, and the relative acquisition voltage differences and substrate CSRs were used to calibrate SiOx CSRs. The blue region denotes the typical CSRs observed from the $\mathrm{Si}<100\rangle$ substrate, after successful analysis through the $120 \mathrm{~nm} \mathrm{SiOx}$. The orange region denotes the measured SiOx CSRs and their estimated relative fields. The purple circles originate from a separate experiment of single bulk SiOx sample that was analyzed under a variety of conditions, consequently changing the applied voltage and CSR. The relative voltage/field of those sets of trials were scaled to best follow the trend established by the $120 \mathrm{~nm} \mathrm{SiOx}$ data. 